INPLASY

PROTOCOL

To cite: Li et al. Tuina for Knee Osteoarthritis Protocol for a systematic review and metaanalysis. Inplasy protocol 202170087. doi:

10.37766/inplasy2021.7.0087

Received: 27 July 2021

Published: 27 July 2021

Corresponding author: Hong Zhang

zhanghongdoctor@sina.com

Author Affiliation:

Rehabilitation Department, Yueyang Hospital of Integrated Traditional Chinese and Western Medicine, Shanghai University of Traditional Chinese Medicine.

Support: Shanghai university of TCM.

Review Stage at time of this submission: Piloting of the study selection process.

Conflicts of interest:

None declared.

\section{Tuina for Knee Osteoarthritis Protocol for a systematic review and meta-analysis}

Li, X1; Zhang, G2; Zhu, J3; Tao, J4; Zhang, S5; Zhang, H6.

Review question / Objective: Knee osteoarthritis (KOA) is a common refractory disease in the worldwide. The main symptoms are characteristic for painful and dysfunction, which is persistent and duration. Tuina is a cost-effective treatment that has potential as a knee osteoarthritis therapy. This protocol is to provide the methods used to assess the effectiveness and safety of tuina for the treatment of patients withKOA.

Condition being studied: Knee osteoarthritis is a common disease, and the Shanghai University of Traditional Chinese Medicine Institute of Chinese and Western Medicine and the Yueyang Hospital of Integrated Traditional Chinese and Western Medicine have been engaged in clinical and evidence-based research on osteoarthritis. Tuina is one of the representatives of non-drug treatments of Chinese medicine for KOA. Clinical research is more common, but the overall research quality has yet to be further clarified, so we plan this program. At the same time, our protocol has successfully reached the stage of study selection process.

INPLASY registration number: This protocol was registered with the International Platform of Registered Systematic Review and Meta-Analysis Protocols (INPLASY) on 27 July 2021 and was last updated on 27 July 2021 (registration number INPLASY202170087).

\section{INTRODUCTION}

Review question / Objective: Knee osteoarthritis (KOA) is a common refractory disease in the worldwide. The main symptoms are characteristic for painful and dysfunction, which is persistent and duration. Tuina is a cost-effective treatment that has potential as a knee osteoarthritis therapy. This protocol is to provide the methods used to assess the 
effectiveness and safety of tuina for the treatment of patients withKOA.

Condition being studied: Knee osteoarthritis is a common disease, and the Shanghai University of Traditional Chinese Medicine Institute of Chinese and Western Medicine and the Yueyang Hospital of Integrated Traditional Chinese and Western Medicine have been engaged in clinical and evidence-based research on osteoarthritis. Tuina is one of the representatives of non-drug treatments of Chinese medicine for KOA. Clinical research is more common, but the overall research quality has yet to be further clarified, so we plan this program. At the same time, our protocol has successfully reached the stage of study selection process.

\section{METHODS}

Participant or population: We will include studies on patients diagnosed with KOA24 regardless of sex, age, race, or severity and duration of disease. Patients with KOA caused by infection, metastatic disease, tumor or fracture are excluded. Patients with KOA associated with serious illnesses such as cancer, liver disease, or kidney disease are also excluded.

Intervention: We will include studies using Tuina as the sole experimental intervention.

Comparator: For control interventions, we will include medication, physical therapy, behavioral therapy, or acupuncture and so on. If other therapies are both used in the experimental and control groups, then the combination of tuina and other therapies will be included. In addition, there is no limitation to the intervention duration and frequency.

Study designs to be included: We will include only randomized controlled trials (RCTs) of tuina for KOA in the treatment groups. Whether or not the expression "randomization" is mentioned with the randomization methods, it will be included in this review. Whether or not the expression "randomization" is mentioned with the randomization methods, it will be included in this review.

Eligibility criteria: Other designs such as animal experiment, case reports, and retrospective studies will be excluded.

Information sources: The following databases will be searched comprehensively from the construction to March 1 2019. It includes five English databases, that is, Medline, PubMed, Cochrane Central Register of Controlled Trials (CENTRAL), EMBASE, and Allied and Complementary Medicine Database (CINAHL). At the same time, three Chinese databases should be involved, which is China National Knowledge Infrastructure (CNKI), Wanfang Data (Wan fang), and VIP Information (VIP). In addition, unpunblished conference proceedings and doctoral and Master's thesis will be collected to ensure the search scope. We No will not restrict the literature language.

Main outcome(s): (1) KOA symptom will be assessed by the Visual Analog Scale (VAS) (0-10) (2) the functional outcomes will be measured by the he Western Ontario and McMaster Universities Osteoarthritis Index (WOMAC).

Additional outcome(s): (1) Quality of life will be measured by the 36-item ShortForm Health Survey (SF-36). (2) Adverse events.

Quality assessment / Risk of bias analysis: Two independent reviewers (Xing $\mathrm{Li}$ and Guohui Zhang) will separately assess methodological quality utilizing the Cochrane risk of bias tool27, Which include the bellowing option: sequence generation; allocation concealment; blinding of participants; blinding of outcome assessment; incomplete outcome data; selective outcome reporting; and other issues. According to these areas, each trial will be classified into low risk, high risk and ambiguous risk respectively. Any disagreement will be negotiated with the third author to achieve consulation. 
Strategy of data synthesis: Review Manager version 5.3 software will be used to execute the data analysis and synthesis.But, heterogeneity will be evaluated by 12 statistic before the data synthesis. In the meta-analyses, we will use a random effects model when the heterogeneity is significant ( $12 \geq 50 \%$ ), while a fixed effects model will be used when the heterogeneity is non-significant(12 $<50 \%)$. Standardized mean differences (SMDs) and the corresponding $95 \%$ confidence interval (Cls) will be used for continuous data (eg, VAS, WOMAC, and SF-36 scores).

Subgroup analysis: If the necessary data are available, we will conduct a subgroup analysis according to the following criteria: (1) the treatment period, (2) different acupuncture points with tuina (3) different types of manipulation (eg, kneading, rolling, pressing).

Sensitivity analysis: We can exclude the studies to identify the robustness of the meta-analysis, which with high risks of bias or outcomes that are seriously distant from the rest of the data. This evaluation refers to sensitivity analyses.

Language: Chinese and English.

Country(ies) involved: China.

Keywords: knee osteoarthritis, protocol, systematic review, tuina.

Dissemination plans: The findings will be disseminated through conference presentations and publication in a peerreviewed, scientific journal.

Contributions of each author:

Author 1 - Xing Li.

Email: 519616533@qq.com

Author 2 - Guohui Zhang.

Email: yyyyzgh827@126.com

Author 3 - Jian Zhu.

Email: 2621166922@qq.com

Author 4 - Jiming Tao.

Email: taoyecheng@163.com

Author 5 - Shuaipan Zhang.

Email: 18939061729@163.com
Author 6 - Hong Zhang.

Email: zhanghongdoctor@sina.com 